\title{
Higher serum levels of tumour necrosis factor and its soluble receptors are associated with ovarian tumours
}

Aleksandra Mielczarek-Palacz, Zdzisława Kondera-Anasz, Justyna Sikora

Department of Immunology and Serology, Medical University of Silesia, Katowice, Poland

Submitted: 26 October 2011

Accepted: 14 January 2012

Arch Med Sci 2012; 8, 5: 848-853

DOI: $10.5114 /$ aoms.2012.31384

Copyright @ 2012 Termedia \& Banach

\section{Abstract}

Introduction: Tumour necrosis factor (TNF) and its soluble receptors type 1 (sTNF-R1) and type 2 (sTNF-R2) have been suggested as key mediators between apoptosis and cancer cell progression. The aim was to examine concentrations of the parameters in the serum of women with ovarian tumour and in the fluid from ovarian cysts of women with serous cystadenoma.

Material and methods: The study included 125 women with ovarian tumours. As a control, sera were obtained from 70 healthy female volunteers. Concentrations of TNF, STNF-R1 and STNF-R2 were measured by enzyme-linked immunosorbent assay (ELISA).

Results: Significant increases of TNF, STNF-R1 and sTNF-R2 were found in the serum of women with ovarian tumour in comparison to the control $(p<0.0001)$. The highest levels of all studied parameters were observed in women with ovarian cancer. In the ovarian cyst fluid the concentrations of the evaluated parameters increased significantly as compared to the serum $(p<0.0001)$.

Conclusions: Our data showed changes in regulatory mechanisms of apoptosis in women with ovarian tumours which are associated with increased concentrations of all studied factors. Serum estimated TNF and especially STNF-R may be used as complementary diagnostic markers in patients with ovarian tumours.

Key words: tumour necrosis factor, soluble receptor of tumour necrosis factor, ovarian tumours.

\section{Introduction}

Ovarian cancer is one of the most lethal gynaecological cancers and a high mortality rate makes this disease a major health problem for women. Approximately $70 \%$ of patients with ovarian cancer are diagnosed at advanced stages (International Federation of Gynecology and Obstetrics - FIGO stage III and IV) and the 5-year survival rate for this group is only $10 \%$ to $40 \%$ [1]. Nevertheless, the still unclear aetiology of ovarian cancer and unclear understanding of the nature of its precursor lesions are the main reasons for the slow development of effective early detection markers and in consequence ineffective targeted therapy.

Recent studies have noted impairments of apoptosis in malignant cells including ovarian cancer cells [2]. Factors of the tumour necrosis factor (TNF) superfamily play an important role in the control of apoptosis. One of the major systems involved in such processes is TNF and its receptors.

The TNF, a protein consisting of 157 amino acids, is synthesized as a membrane-anchored $26-\mathrm{kDa}$ precursor (pro-TNF) that is cleaved to the
Corresponding author: Dr Aleksandra Mielczarek-Palacz Department of Immunology and Serology

Medical University of Silesia 15 Raciborska 40-074 Katowice, Poland Phone: +48322087412 Fax: +48 322087438 E-mail: apalacz@sum.edu.pl 
secreted 17-kDa form [3]. In response to TNF, cell activation is mediated via two TNF receptors (TNF-R): tumour necrosis receptor type 1 (TNF-1R1) and tumour necrosis receptor type 2 (TNF-R2) [4]. TNF-R1, with $55 \mathrm{kDa}$ molecular weight, is the main receptor mediating the cellular effects of TNF in most cell type [5]. It belongs to the death receptor family and contains the death domain (DD), that shares the capability of cell activation to independent metabolic pathways. TNF-R2, which is identified as a $75 \mathrm{kDa}$ protein, does not possess a DD, although it can mediate a cell death signal, which may be indirect through TNF-R1 [6]. Both receptors are ubiquitously expressed, whereas TNF-R2 is mainly expressed on immune cells [7]. The binding of homotrimeric TNF to either receptor can activate three important signalling pathways: TNF receptor associated death domain (TRADD); nuclear factor $\kappa \mathrm{B}(\mathrm{NF}-\kappa \mathrm{B})$, a major cell survival signal; and c-Jun $\mathrm{N}$-terminal kinase (JNK) signalling cascades [8].

The membrane-bound receptors can be cleaved and released as a soluble form from the cell surface following proteolysis as the soluble TNF-R1 (sTNF-R1) and the soluble TNF-R2 (sTNF-R2). Increased concentrations of TNF and its soluble receptors in serum and other biological fluids are associated with different diseases, including malignant diseases [9]. For this reason, the aim of the study was to evaluate the selected mechanisms which control apoptosis, through measurement of the concentrations of TNF, STNF-R1 and STNF-R2 in the pre-treatment serum of affected women and in ovarian cyst fluid of women with serous cystadenoma.

\section{Material and methods}

\section{Patients and clinical samples}

The study group consisted of 125 women aged 21 to 62 years (mean age: $46.8 \pm 11.7$ years) with newly diagnosed ovarian tumour. This group included 50 women aged 21-62 years (mean age: $44.2 \pm 11.5$ years) with ovarian serous cystadenoma, 35 women aged
24 to 64 years (mean age: $43.9 \pm 7.2$ years) with mature cystic teratoma, and 40 women aged 29 to 59 years (mean age: $45.5 \pm 10.4$ years) with ovarian serous cystadenocarcinoma in stage la. The tumour assessment was based on results of clinical symptoms, gynaecological and histological examinations and laboratory analysis, including CA125 level. They did not have any sign of endometriosis previously. The tumours were graded according to World Health Organization criteria and staging was done by implementing the criteria of FIGO. All women were patients of the Department of Gynaecology and Obstetrics of the $6^{\text {th }}$ Hospital in Katowice, Poland. The control group consisted of 70 healthy female volunteers aged $21-62$ years (mean age: $46.9 \pm 9.2$ years), who had no evidence of pathological disorders or any inflammations in the reproductive system. Not all women smoked and none had received hormonal or anti-inflammatory treatment during the 3 months preceding material collection or surgery. Characteristics of the study population are shown in Table I.

The analysed material was venous peripheral blood obtained by venipuncture from all studied women and the ovarian cyst fluid of women with serous cystadenoma. In women with ovarian tumour, the blood was taken after establishing the diagnosis, directly prior to the operation, and the ovarian cyst fluid was obtained during the surgery. In the control group, the blood was taken when the women were admitted for follow-ups. Blood and fluid was centrifuged at $600 \times \mathrm{g}$ for $10 \mathrm{~min}$ and next samples were aliquoted and stored at $-70^{\circ} \mathrm{C}$ until the time for measurement.

\section{Cytokine assay}

The concentrations of TNF, TNF-R1 and TNF-R2 were determined by enzyme-linked immunosorbent assay (ELISA) using the commercial kit Bender MedSystems (Vienna, Austria). All determinations were performed in duplicate, according to the instructions. The sensitivity of the kits was $5.8 \mathrm{pg} / \mathrm{ml}$ for TNF, $80 \mathrm{pg} / \mathrm{ml}$ for sTNF-R1 and $0.15 \mathrm{pg} / \mathrm{ml}$ for sTNF-R2. CA125 level in serum of all studied women

Table I. Baseline characteristics of women with ovarian tumours and control group

\begin{tabular}{|lcc|}
\hline Variable & Ovarian tumour & Control group \\
\hline Number of women & 125 & 70 \\
\hline Age $[$ years] & $21-62(46.8 \pm 11.7)$ & $21-62(46.9 \pm 9.2)$ \\
\hline BMI $\left[\mathrm{kg} / \mathrm{m}^{2}\right]$ & $18-28(25.7 \pm 3.9)$ & $21-32(26.1 \pm 3.6)$ \\
\hline $\begin{array}{l}\text { Menstrual status: } \\
\text { Pre-menopausal }\end{array}$ & 68 & 30 \\
\hline Post-menopausal & 57 & 30 \\
\hline \begin{tabular}{l} 
Menstrual phase: \\
\hline Follicular phase
\end{tabular} & 68 & 40 \\
\hline Luteal phase & $>35$ & $<35$ \\
\hline Concentration of CA125 [U/ml] & & 30 \\
\hline
\end{tabular}

Values are means $\pm S D$. BMI-body mass index 
was measured using a commercial Microparticle Enzyme Immunosorbent Assay (MEIA) kit (Abbott Diagnostics, Wiesbaden, Germany). The upper reference limit for CA125 was used according to the manufacturer's recommendation - below $35 \mathrm{U} / \mathrm{ml}$.

The Ethical Committee of the Medical University of Silesia according to the Declaration of Helsinki approved this study.

\section{Statistical analysis}

Results of TNF, sTNF-R1 and STNF-R2 are presented as mean \pm standard deviation and were examined for normality of distribution by the ShapiroWilk test. Parametric data were analysed using a Student's $t$-test. For nonparametric data, Fisher's exact test was used to indicate statistical significance because it analyses the variance relationship both within and among the groups. The data of CA125 level are presented as the number of women with higher or lower concentration than $35 \mathrm{U} / \mathrm{ml}$. Correlations were tested by Spearman's rank correlation test and presented as the correlation coefficient $(r)$. A $p<0.05$ was considered statistically significant. All analyses were performed with Statistica for Windows 8.0 software.

\section{Results}

Concentrations of TNF, sTNF-R1 and sTNF-R2 in the serum of women with ovarian tumours and the control group and also in the ovarian cyst fluid are shown in Table II.

\section{TNF}

Mean serum TNF level of women with ovarian tumours was significantly higher compared to controls. Along with higher tumour stage the concentration of TNF increased. The highest studied pa- rameter levels were observed in serum of women with ovarian cancer. Differences between all groups were statistically significant: $p<0.01$ between women with ovarian serous cystadenoma and mature cystic teratoma; $p<0.0001$ between women with ovarian serous cystadenoma and ovarian cancer; $p<0.0001$ between mature cystic teratoma and ovarian cancer. Additionally, concentration of TNF was significantly lower in serum compared to ovarian cyst fluid $(p<0.0001)$.

\section{STNF-R1}

Serum sTNF-R1 level was significantly higher in women with ovarian tumours compared to controls ( $p<0.0001$ ). Along with higher tumour stage the concentration of receptor increased, but the difference between STNF-R1 level in women with ovarian serous cystadenoma and mature cystic teratoma was not statistically significant. The highest concentration of sTNF-R1 was observed in serum of women with ovarian cancer, and it was significantly higher compared to other studied groups ( $p<0.0001)$. Increased STNF-R1 in ovarian cyst fluid in comparison with serum of women with ovarian serous cystadenoma $(p<0.0001)$ was observed.

\section{sTNF-R2}

Serum sTNF-R2 level was significantly higher in women with ovarian tumours compared to controls $(p<0.0001)$. Along with higher tumour stage the concentration of receptor also increased, but the difference between STNF-R2 level in women with ovarian serous cystadenoma and mature cystic teratoma was not statistically significant. The highest concentration of STNF-R2 was observed in serum of women with ovarian cancer, and it was significantly higher compared to other studied groups $(p<0.0001)$. Increased sTNF-R2 in ovarian cyst fluid

Table II. Serum levels of TNF, TNF-R1 and TNF-R2 of women with ovarian tumors and in ovarian cyst fluid of women with serous cystadenoma

\begin{tabular}{|c|c|c|c|c|c|c|c|}
\hline \multirow[t]{2}{*}{ Group } & \multirow[t]{2}{*}{$n$} & \multicolumn{2}{|c|}{$\mathrm{TNF}[\mathrm{pg} / \mathrm{ml}]$} & \multicolumn{2}{|c|}{ TNF-R1 [ng/ml] } & \multicolumn{2}{|c|}{ TNF-R2 [ng/ml] } \\
\hline & & Range & Mean \pm SD & Range & Mean \pm SD & Range & Mean \pm SD \\
\hline \multicolumn{8}{|l|}{ Serum: } \\
\hline Ovarian tumors & 125 & $15.57-48.90$ & $24.90 \pm 7.91^{\star}$ & $1.01-2.60$ & $1.71 \pm 0.53^{*}$ & $2.40-5.30$ & $3.51 \pm 0.74^{\star}$ \\
\hline Serous cystadenoma & 50 & $15.57-25.18$ & $18.59 \pm 2.70^{*, t, \pm, \S}$ & $1.01-1.53$ & $1.25 \pm 0.15^{*,+, \S, \|}$ & $2.46-3.73$ & $3.08 \pm 0.33^{*, \pm, * *}$ \\
\hline Mature teratoma & 35 & $16.45-25.11$ & $22.94 \pm 2.30^{*, \neq}$ & $1.38-2.45$ & $1.82 \pm 0.32^{*, \neq}$ & $2.40-3.88$ & $3.19 \pm 0.56^{*, \neq}$ \\
\hline Serous cystadenocarcinoma & 40 & $24.72-48.93$ & $34.12 \pm 6.10^{*}$ & $1.99-2.63$ & $2.33 \pm 0.19^{*}$ & $3.17-5.35$ & $4.28 \pm 0.63^{\star}$ \\
\hline Control & 70 & $<5.8$ & $<5.8$ & $0.59-0.68$ & $0.62 \pm 0.04$ & $1.10-2.12$ & $1.80 \pm 0.41$ \\
\hline \multicolumn{8}{|l|}{ Ovarian cyst fluid: } \\
\hline Serous cystadenoma & 50 & $40.29-60.55$ & $50.97 \pm 5.79$ & $2.79-4.25$ & $3.58 \pm 0.43$ & $4.1-6.33$ & $5.34 \pm 0.61$ \\
\hline
\end{tabular}

Values are means $\pm S D ;{ }^{*} p<0.0001$ compared to control group, ${ }^{\dagger} p<0.01$ in mpared to mature teratoma, $\neq p<0.0001$ compared to ovarian cancer; $s p<0.0001$ compared to ovarian cyst fluid of serous cystadenoma, $\| p<0.0001$ compared to mature teratoma, ${ }^{* *} p<0.001$ compared to ovarian cyst fluid of serous cystadenoma 
in comparison with serum of women with ovarian serous cystadenoma $(p<0.0001)$ was observed.

A non-statistically significant correlation between levels of studied parameters in serum and in fluid from ovarian cyst was observed. Additionally, a statistically significant positive correlation was found between the concentration of TNF and STNF-R1 $(r=0.75, p<0.0001)$ and the concentration of TNF and STNF-R2 $(r=0.77, p<0.0001)$.

In addition we analysed the relationship between TNF and CA125. Positive significant correlations between TNF and CA125 ( $r=0.747, p<0.0001)$, between sTNF-R1 and CA125 $(r=0.842, p<0.0001)$ and between sTNF-R2 and CA125 $(r=0.662, p<0.001)$ were observed.

\section{Discussion}

Ovarian cancer is generally diagnosed at advanced stages of the disease. Moreover, the fact that the survival of the patients with stages I-II of the disease ranges from $60 \%$ to $90 \%$ suggests the potential for a high treatment rate with earlier detection of the disease. That fact is associated with finding specific markers of ovarian tumours. Strategies for identification of biomarkers for non-invasive tests, such as ELISA assays, have been in use for decades. Unfortunately, neither aetiology nor pathogenesis of ovarian tumours is still completely known even though there is intensive research of the disease. A better knowledge of the disease has led to the study of newer biological or molecular prognostic factors that may complement or even replace some of the less objective convectional parameters.

It is known that the TNF-TNF-R system plays an important role in inflammation, angiogenesis, programmed cell death, and proliferation, which are all crucial components in malignant transformations. The complicated roles of TNF in cancer development have emerged. Its anticancer property is mainly through inducing cancer death. However, TNF also stimulates proliferation, survival, migration and angiogenesis in most tumours that are resistant to TNF-induced cytotoxicity, resulting in tumour promotion. TNF is a double-edged sword that could be either pro- or anti-tumourigenic. Moreover, soluble forms of TNF-R1 and TNF-R2 are derived from the extracellular binding domains of each receptor and they regulate the activity of TNF by competing with its receptors. Some research supports laboratory results and strongly suggests that TNF and its soluble receptors could be useful in cancer detection and staging or predicting prognosis [10].

In this study, our results did not show the presence of TNF in the serum of the control. However, increased concentration of it was associated with the clinical extent of the tumours. The highest TNF level was observed in the serum of women with ovarian cancer. We also evaluated the concentration of TNF in the ovarian cyst fluid of women with ovarian serous cystadenoma in comparison to the serum. Significantly higher levels of this parameter may indicate a local generated immune response and intensity of cancerogenesis. Moreover, it could be the reason for destruction of the peritoneal-like epithelium, which builds the cyst.

Studies have indicated significant changes of TNF level in women with ovarian tumours but the results are still ambiguous. The majority of them observed significant increases in TNF level in the serum of women with ovarian cancer [11-14]. Their investigation first of all related to epithelial ovarian cancer. Dobrzycka et al. [11] apart from increased TNF level also found a correlation with tumour stage and with reduced mean survival time. On the other hand, Kutteh et al. [15] observed the highest TNF level in women with papillary serous cystadenocarcinoma and the lowest in women with mucinous cystadenomas. Naylor et al. [16] reported higher expression of TNF in ovarian cancer cells, which was dependent on the stage of progression of disease. This may confirm our results of higher level of TNF in ovarian cyst fluid and the suggested local immune response against cancer cells. However, other studies did not detect TNF in the serum of women with ovarian cancer, in women with non-malignant ovarian cysts or in bioptates of ovarian fibrosarcoma, which indicated that the lower TNF secretion by immune cells might suppress the influence on lymphocyte T proliferation [17, 18].

In our study, we additionally examined the concentration of STNF receptors in the serum of affected women. In controls STNF-R1 and sTNF-R2 levels were in the range of physiological values. At the same time, in the group of women with ovarian tumour concentrations of both receptors significantly exceeded the range of physiological values. Our results indicated the highest STNF-R1 and STNF-R2 in women with ovarian cancer and the lowest in women with ovarian serous cystadenoma. This suggested that both STNF-R1 and STNF-R2 levels can be useful as laboratory markers for differential diagnostics of ovarian tumours. We also affirmed that in women with ovarian tumour, the secretion of STNF-R2 in the serum was higher than STNF-R1. Moreover, a strong positive correlation between TNF or its receptors and CA125 might suggest that CA125, being a tumour marker, is closely related to the cytokine system. TNF correlated with severity of cancer, indicating its superiority as a marker for further, larger studies, but the measurement of complementary serum markers, such as TNF, can improve the use of marker screening for ovarian tumour. Studies suggested that other tumour markers have also been implicated in pathogenesis of ovarian tumours, but their diagnostic use is limited [19]. 
This observation was consistent with the results of other authors [20-23]. In addition, the ovarian cancer cells had the capacity for spontaneous secretion of more sTNF-R1, but less receptor type 2 [24]. Additionally, serum TNF-R1 level correlated with morphological ultrasound score and CA125 [22]. The observations made in Burger's study [25] showed a relationship between levels of CA125, sTNF-R1 and sTNF-R2 and risk of progression of epithelial ovarian malignancies. Among patients with low or high CA125 levels, those with high sTNF-R1 levels and low sTNF-R2 levels had the lowest risk, patients with low-low or high-high sTNF-R1 and sTNF-R2 levels, respectively, had an intermediate risk, and patients with low STNF-R1 levels and high sTNF-R2 levels had the highest risk of progression [25].

The present study demonstrated higher concentration of STNF-R1 and sTNF-R2 in the fluid than in the serum of affected women and suggested intensity of the local immune response. The higher receptor levels might be associated with augmented secretion of soluble receptors by the malignant cells. It leads to a decrease in count of membrane type receptors on the cell surface and prevents interaction between TNF and its membrane receptors [26] and, in consequence, leads to inhibition of apoptosis induction. In that way, malignant cells can protect themselves from the immune reaction. Analysis of cytokine levels of ovarian cyst fluid may be a pure way to study cytokine expression to gain more insight into tumour-host interaction. Studies indicate that higher IL-6 or IL-8 levels in cyst fluid are correlated with malignancy and are observed in malignant cyst fluids. Additionally, T helper 1 (Th1) subtype and Th2 cytokine expression depends on the type of tumours [27]. In our results, higher levels of TNF and its receptors in cyst fluid than in serum confirmed that the immunosuppressive state created by ovarian tumour is reflected in the cystic fluid within the tumour.

An interesting observation was made in our analysis of the correlation between TNF and its receptor levels in the serum of women with ovarian tumours. A significant positive correlation between TNF and TNF-R1, as well as TNF and TNF-R2, which we observed, may suggest the ovarian cancer cells' capacities for autocrine TNF secretion with contemporary shedding of both receptors from the cell surface. Similar results were reported by Langkopf et al. [28], who suggest that higher levels of TNF and its receptors in biological fluids are the ovarian cancer cells' protection against cytotoxic TNF properties. Given the suggested pro-apoptotic role of TNF, elevated concentrations of TNF-R may antagonize TNF in the environment, and consequently attenuate the apoptotic process of inflexed ovarian cancer cells. It has been suggested that the failure to eliminate ovarian cancer cells, possibly due to abnormal- ities of the immune system, especially impairment of the TNF-sTNF-R pathway, may be conducive to the development of cancer. Moreover, the mechanisms regulating STNF-R in women with ovarian cancer are unknown. Because of competitive TNF-sTNF-R binding, soluble receptors serve as TNF antagonist. It has been suggested that an increase in circulating sTNF-R level in women with ovarian cancer may promote survival of ovarian cancer cells by antagonizing TNF, a pro-apoptotic cytokine.

Impairments of the TNF-TNF-R system are observed in other gynaecological diseases, especially in endometriosis and endometriosis-associated infertility [29, 30]. Increased serum and peritoneal fluid TNF levels have been implicated in the pathophysiology of endometriosis. Because of this, higher TNF may indicate both conditions - endometriosis and ovarian tumours. However, in women with endometriosis, TNF acts generally as a pro-inflammatory cytokine rather than as a pro-apoptotic factor. It can increase the permeability of local blood vessels, thereby causing inflammatory exudation into the peritoneal cavity and aggravation of peritoneal inflammation, and in consequence reduces fertility [30].

In conclusion, among women with ovarian tumours, impairment of apoptosis appears to occur, which demonstrates the increase in concentrations of all the studied parameters. Serum TNF, sTNF-R1 and STNF-R2 levels are more elevated in patients with ovarian cancer. Higher concentrations of them in the ovarian cyst fluid than in serum suggest local suppression of the immune response, and intensity of apoptosis impairment. Serum TNF, sTNF-R1 and STNF-R2 are all biochemical factors which may be implicated in the pathophysiology of ovarian cancer. Their levels, especially sTNF-R1 and sTNF-R2, might be used as complementary diagnostic markers in patients with ovarian tumours, and may gradually improve early diagnostics for women with ovarian cancer.

\section{References}

1. Kwong J, Chan FL, Wong KK, et al. Inflammatory cytokine tumor necrosis factor alpha confers precancerous phenotype in an organoid model of normal human ovarian surface epithelial cells. Neoplasia 2009; 11: 529-41.

2. Fulda S. Cell death in hematological tumors. Apoptosis 2009; 14: 409-23.

3. Wang $X$, Lin Y. Tumor necrosis factor and cancer, buddies or foes? Acta Pharmacol Sin 2008; 29: 1275-88.

4. So T, Lee SW, Croft M. Tumor necrosis factor/tumor necrosis factor receptor family members that positively regulate immunity. Int J Hematol 2006; 83: 1-11.

5. Ihnatko R, Kubes M. TNF signaling: early events and phosphorylation. Gen Physiol Biophys 2007; 26: 159-67.

6. Popivanova BK, Kitamura K, Wu Y, et al. Blocking TNF-alpha in mice reduces colorectal carcinogenesis associated with chronic colitis. J Clin Invest 2008; 118: 560-70.

7. Aggarwal BB. Signalling pathways of the TNF superfamily: a double-edged sword. Nat Rev Immunol 2003; 3: 745-56. 
8. Shen HM, Pervaiz S. TNF receptor superfamily-induced cell death: redox-dependent execution. FASEB J 2006; 20: 1589-98.

9. Balkwill F. Tumour necrosis factor and cancer. Nat Rev Cancer 2009; 9: 361-71.

10. Rzymski P. Tumor necrosis fFactor alpha receptors p55 and p75 and ovarian cancer - state-of-the-art research and clinical implications. Arch Med Sci 2005; 1: 3-7.

11. Dobrzycka B, Terlikowski SJ, Kowalczuk O, Kinalski M. Circulating levels of TNF-alpha and its soluble receptors in the plasma of patients with epithelial ovarian cancer. Eur Cytokine Netw 2009; 20: 131-4.

12. Yigit R, Figdor CG, Zusterzeel PL, Pots JM, Torensma R, Massuger LF. Cytokine analysis as a tool to understand tumourhost interaction in ovarian cancer. Eur J Cancer 2011; 47: 1883-9.

13. Gadducci A, Ferdeghini M, Castellani C, et al. Serum levels of tumor necrosis factor (TNF), soluble receptors for TNF (55- and 75-kDa sTNFr), and soluble CD14 (sCD14) in epithelial ovarian cancer. Gynecol Oncol 1995; 58: 184-8.

14. Maccio A, Lai P, Santona MC, Pagliara L, Melis GB, Mantovani G. High serum levels of soluble IL-2 receptor, cytokines, and $C$ reactive protein correlate with impairment of $T$ cell response in patients with advanced epithelial ovarian cancer. Gynecol Oncol 1998; 69: 248-52.

15. Kutteh WH, Kutteh CC. Quantitation of tumor necrosis factor-alpha, interleukin-1 beta, and interleukin- 6 in the effusions of ovarian epithelial neoplasms. Am J Obstet Gynecol 1992; 167: 1864-9.

16. Naylor MS, Stamp GW, Foulkes WD, Eccles D, Balkwill FR. Tumor necrosis factor and its receptors in human ovarian cancer. Potential role in disease progression. J Clin Invest 1993; 91: 2194-206.

17. Fukuda T, Yamaguchi T, Yamaki T, Suzuki T. Ovarian fibrosarcoma producing multiple cytokines. Pathol Int 2001; 51: 739-43.

18. Nowak M, Glowacka E, Szpakowski M, et al. Proinflammatory and immunosuppressive serum, ascites and cyst fluid cytokines in patients with early and advanced ovarian cancer and benign ovarian tumors. Neuro Endocrinol Lett 2010; 31: 375-83.

19. Gadducci A, Tana R, Cosio S, Genazzani AR. The serum assay of tumour markers in the prognostic evaluation, treatment monitoring and follow-up of patients with cervical cancer: a review of the literature. Crit Rev Oncol Hematol 2008; 66 : 10-20.

20. Kulbe H, Thompson R, Wilson JL, et al. The inflammatory cytokine tumor necrosis factor-alpha generates an autocrine tumor-promoting network in epithelial ovarian cancer cells. Cancer Res 2007; 67: 585-92.

21. Grosen EA, Granger GA, Gatanaga M, et al. Measurement of the soluble membrane receptors for tumor necrosis factor and lymphotoxin in the sera of patients with gynecologic malignancy. Gynecol Oncol 1993; 50: 68-77.

22. Rzymski P, Opala T, Wilczak M, Woźniak J, Sajdak S. Serum tumor necrosis factor alpha receptors p55/p75 ratio and ovarian cancer detection. Int J Gynaecol Obstet 2005; 88: 292-8.

23. Opala T, Rzymski P, Wilczak M, Woźniak J. Evaluation of soluble tumour necrosis factor alpha receptors p55 and p75 in ovarian cancer patients. Eur J Gynaecol Oncol 2005; 26: 43-6.

24. Gatanaga M, Grosen EA, Burger RA, Granger GA, Gatanaga T. Release of soluble TNF/LT receptors from a human ovarian tumor cell line (PA-1) by stimulation with cytokines in vitro. Lymphokine Cytokine Res 1993; 12: 249-53.

25. Burger RA, Darcy KM, DiSaia PJ, et al. Association between serum levels of soluble tumor necrosis factor receptors/CA
125 and disease progression in patients with epithelial ovarian malignancy: a gynecologic oncology group study. Cancer 2004; 101: 106-15.

26. Szlosarek PW, Grimshaw MJ, Wilbanks GD, et al. Aberrant regulation of argininosuccinate synthetase by TNF-alpha in human epithelial ovarian cancer. Int J Cancer 2007; 121: 6-11.

27. Yigit R, Massuger LF, Zusterzeel PL, Pots J, Figdor CG, Torensma R. Cytokine profiles in cyst fluids from ovarian tumors reflect immunosuppressive state of the tumor. Int J Gynecol Cancer 2011; 21: 1241-7.

28. Langkopf F, Atzpodien J. Soluble tumour necrosis factor receptors as prognostic factors in cancer patients. Lancet 1994; 344: 57-8.

29. Buks J, Wilczak M, Rzymski P, Opala T. Do soluble p55 and p75 TNF-alpha receptor concentrations play a role in women with primary sterility? Arch Med Sci 2010; 6: 264-9.

30. Tao Y, Zhang O, Huang W, Zhu H, Zhang D, Luo W. The peritoneal leptin, MCP-1 and TNF-alpha in the pathogenesis of endometriosis-associated infertility. Am J Reprod Immunol 2011; 65: 403-6. 